\title{
The role of NCAM in auditory fear conditioning and its modulation by stress: a focus on the amygdala
}

\section{R. Bisaz and C. Sandi*}

Laboratory of Behavioral Genetics, Brain Mind Institute, Ecole Polytechnique Federale de Lausanne (EPFL), 1015 Lausanne, Switzerland

${ }^{*}$ Corresponding author: Prof. C. Sandi, Brain Mind Institute, Ecole Polytechnique Federale de Lausanne (EPFL), Station 19, 1015 Lausanne, Switzerland. E-mail: carmen.sandi@epfl.ch

Chronic stress in rodents was shown to induce structural shrinkage and functional alterations in the hippocampus that were linked to spatial memory impairments. Effects of chronic stress on the amygdala have been linked to a facilitation of fear conditioning. Although the underlying molecular mechanisms are still poorly understood, increasing evidence highlights the neural cell adhesion molecule (NCAM) as an important molecular mediator of stress-induced structural and functional alterations. In this study, we investigated whether altered NCAM expression levels in the amygdala might be related to stress-induced enhancement of auditory fear conditioning and anxiety-like behavior. In adult C57BL/6J wild-type mice, chronic unpredictable stress resulted in an isoform-specific increase of NCAM expression (NCAM-140 and NCAM-180) in the amygdala, as well as enhanced auditory fear conditioning and anxietylike behavior. Strikingly, forebrain-specific conditional NCAM-deficient mice (NCAM-floxed mice that express the cre-recombinase under the control of the promoter of the $\alpha$-subunit of the calcium-calmodulin-dependent protein kinase II), whose amygdala NCAM expression levels are reduced, displayed impaired auditory fear conditioning which was not altered following chronic stress exposure. Likewise, chronic stress in these conditional NCAM-deficient mice did not modify NCAM expression levels in the amygdala or hippocampus, while they showed enhanced anxiety-like behavior, questioning the involvement of NCAM in this type of behavior. Together, our results strongly support the involvement of NCAM in the amygdala in the consolidation of auditory fear conditioning and highlight increased NCAM expression in the amygdala among the mechanisms whereby stress facilitates fear conditioning processes.

Keywords: Amygdala, auditory fear conditioning, chronic stress, mice, NCAM, PSA-NCAM

Received 11 September 2009, revised 06 December 2009, accepted for publication 29 December 2009
Chronic stress in rodents was shown to induce structural and functional alterations in the hippocampus and prefrontal cortex, including shortening and debranching of pyramidal neurons (Cerqueira et al. 2007; Cook \& Wellman 2004; Holmes \& Wellman 2009; Magarinos et al. 1997; Radley et al. 2006; Watanabe et al. 1992; Woolley et al. 1990), suppressed long-term potentiation (LTP) (Donohue et al. 2006; Pavlides et al. 2002) and cognitive impairments in a variety of spatial learning and memory tasks (Conrad et al. 1996; Li et al. 2006; Luine et al. 1994; Park et al. 2001; Sandi et al. 2003; Venero et al. 2002). In contrast, in rats, pyramidal and stellate neurons of the basolateral complex of the amygdala (BLA) were reported to exhibit enhanced dendritic branching in response to 10 and 21 days of chronic restrain (but not unpredictable) stress, which was accompanied by increased anxiety-related behaviors (Roozendaal et al. 2009; Vyas \& Chattarji 2004; Vyas et al. 2002; Vyas et al. 2004; Vyas et al. 2006) and potentiated conditioned fear responses (Conrad et al. 1999; Cordero et al. 2003; Sandi et al. 2001). Interestingly, even a single 2-h episode of immobilization stress or acute corticosterone treatment was sufficient to induce a delayed dendritic expansion in the BLA, which was accompanied by increased anxiety-related behaviors (Mitra et al. 2005; Mitra \& Sapolsky 2008).

The neural cell adhesion molecule (NCAM), which has been shown to be downregulated in the hippocampus of rats and mice chronically stressed for 3-4 weeks (Alfonso et al. 2006; Bisaz et al. 2009b; Sandi et al. 2001; Shin et al. 2009; Touyarot \& Sandi 2002; Venero et al. 2002), was proposed to play a key role in these structural and functional alterations (Sandi 2004; Sandi \& Bisaz 2007). NCAM is a glycoprotein of the immunoglobulin superfamily, which through homoand heterophilic binding mediates processes such as neurite outgrowth and fasciculation, and activity-dependent synaptic plasticity in the developing and adult nervous system (Fields \& Itoh 1996; Kiss \& Muller 2001; Maness \& Schachner 2007; Muller et al. 2008). NCAM has been implicated in memory formation (Cambon et al. 2003; Cambon et al. 2004; Conboy et al. 2008; Lopez-Fernandez et al. 2007; Sandi et al. 2004; Venero et al. 2006) and on emotional processes (Conboy et al. 2008). Genetic ablation of NCAM and interventions that interfere with NCAM function (e.g. administration of antibodies or peptides) were shown to result in impaired synaptic plasticity and learning and memory deficits (Conboy et al. 2008; Hartz \& Ronn 2008). In addition, mice with a constitutional ablation of NCAM gene were found to display increased intermale aggression, enhanced anxiety, altered stress responses, and impaired fear conditioning (Senkov et al. 2006; Stork et al. 1997; Stork et al. 2000).

However, it is not yet known whether stress can affect NCAM expression in the amygdala that might 


\section{Bisaz and Sandi}

be connected to stress-induced changes in amygdalarelated behaviors. We aimed here to address these two interrelated questions by first asking whether expression of the major NCAM isoforms in the amygdala might be altered, and whether anxiety-related behaviors and auditorycued fear conditioning responses might be enhanced, as a consequence of chronic stress exposure in C57BL/6J wildtype mice. Second, we assessed the potential implication of reduced NCAM amygdala expression on anxiety and on fear conditioning responses by testing conditional NCAMdeficient mice in which NCAM is ablated in the forebrain by the second postnatal week (Bukalo et al. 2004). Finally, we submitted conditional NCAM-deficient mice to chronic stress and evaluated their subsequent NCAM levels, as well as their auditory fear conditioning and anxiety-like behavior. Our findings suggest a role for NCAM in the amygdala in the consolidation of auditory fear conditioning, as well as the involvement of increased amygdala NCAM expression in stress-induced potentiation of auditory-cued fear memories.

\section{Materials and methods}

\section{Subjects}

Chronic unpredictable stress experiments were conducted on 7to 9-month-old in-house bred C57BL/6J wild-type male mice, originating from eight different breeding couples. Experiments on conditional NCAM-deficient mice were conducted in age-matched adult conditional NCAM-deficient male mice (NCAMffcre) and their control littermates (NCAMff) at 6-8 months of age. The generation of the conditional NCAM-deficient mice has been described previously (Bukalo et al. 2004). Briefly, homozygous NCAM-floxed mice were bred with homozygous NCAM-floxed mice that express the crerecombinase under the control of the promoter of $\alpha$-subunit of the calcium-calmodulin-dependent protein kinase II ( $\alpha$ CaMKII). The progeny was homozygous for the NCAM-floxed alleles, half of them carried the $\alpha$ CaMKII-cre transgene (NCAMffcre) and the rest were control littermates (NCAMff). These mice originated from six different breeding couples, which were previously backcrossed for more than 10 generations into the $\mathrm{C} 57 \mathrm{BL} / 6 \mathrm{~J}$ background. All mice were housed in groups of two to five in standard plastic cages and they were kept under a 12-h light/dark cycle (light on at 0730 h) with ad libitum access to food and water. All the procedures described were conducted in accordance with the Swiss National Institutional Guidelines on Animal Experimentation and approved by the Swiss Cantonal Veterinary Office Committee for Animal Experimentation. The number of animals used in this study was kept to a minimum, as was animal suffering in all procedures.

\section{Chronic stress procedure}

The unpredictable chronic stress protocol involved exposing animals to a daily stressful situation at an unpredictable moment for 28 days (between $0800 \mathrm{~h}$ and $1600 \mathrm{~h}$, with timing randomly distributed over the 28 days). The stress stimuli used each day were one of the following: 6-min tail suspension; $3 \times 0.4 \mathrm{~mA}$ inescapable footshock; 4-h exposure to soiled, damp sawdust; 2 -h exposure on an elevated platform; 1-h immobilization in a plastic tube; 30-min exposure to $16^{\circ} \mathrm{C} ; 2$ days inversed light/dark cycle; 10 -min exposure to an older, aggressive conspecific; 1-h exposure to 2,5-Dihydro-2,4,5trimethylthiazoline (TMT); and 8-h with a $40^{\circ}$ cage inclination. All animals were weighed and the state of their coat was evaluated on a regular basis (every 3-5 days). We performed three chronic stress experiments. In the first one, C57BL/6J wild-type male mice were divided into four groups ( $n=7-11 /$ group) of which two were left undisturbed and two were chronically stressed for 28 days. One of the control groups and a group of chronically stressed animals were used for behavioral studies, while the remaining two served for biochemical analyses. The second chronic stress experiment, which was designed to study anxiety-like behavior after chronic stress, involved C57BL/6J wild-type male mice that were divided into two groups ( $n=8-11 /$ group): one was kept undisturbed and the other one was exposed to the chronic stress procedure for 28 days. The third chronic stress experiment was performed in conditional NCAM-deficient mice that were divided into four groups ( $n=5-6 /$ group) of which two were chronically stressed and the other two remained undisturbed. One of the control groups and a group of chronically stressed animals were used for behavioral analyses, while the remaining two served for biochemical analyses.

\section{Amygdala sample preparation}

NCAM and PSA-NCAM levels in the amygdala were assayed both after chronic stress and in NCAM-deficient mice. In the chronic stress study, chronically stressed and control mice ( $n=8-9$ /group) were decapitated on the morning of day 29. Trunk blood was collected for subsequent quantification of corticosterone levels and the brains were quickly removed and frozen in isopentane and stored at $-80^{\circ}$ until they were further processed. Amygdala and hippocampal fractions (the latter only in a specific experiment) were obtained with tissue punches from $300 \mu \mathrm{m}$ frozen brain slices, according to the protocol from Palkovits (1973). In the mutant mice study, naïve NCAMffcre and NCAMff mice ( $n=4-6 /$ genotype) were sacrificed in the morning and their brains were quickly removed. This tissue was kept on an icecold plate and the amygdala was dissected out rapidly.

From amygdala and hippocampal dissected samples, crude synaptosomal pellets were obtained according to a protocol modified from Lynch and Voss (1991) that has been previously shown to be effective to detect stress-induced changes in NCAM expression (Bisaz et al. 2009b; Touyarot \& Sandi 2002; Touyarot et al. 2004; Venero et al. 2006). In brief, the tissue was homogenized with 20 strokes of a plastic homogenizer in 10 volumes of ice cold HEPES (4 mM) buffered sucrose (0.32 M), containing a freshly added cocktail of protease inhibitors (Complete EDTA-free, Roche Diagnostics $\mathrm{GmbH}$, Mannheim, Germany) and $1 \mathrm{mM}$ ethylenediaminetetraacetic acid (EDTA). The solution was centrifuged at $1000 \boldsymbol{g}$ for $5 \mathrm{~min}$ at $4^{\circ} \mathrm{C}$ and the supernatant was centrifuged at $15000 \mathrm{~g}$ for $15 \mathrm{~min}$, resuspending the resulting pellet in phospate-buffered solution (PBS) and $1 \%$ NP-40, containing protease inhibitors and $1 \mathrm{mM}$ EDTA. The protein concentration for each sample was estimated by the method of Lowry et al. (1951).

\section{Quantitative immunoblotting of NCAM}

Expression levels of three main NCAM isoforms (NCAM-180, NCAM140 and NCAM-120) were measured in immunoblots of crude synaptosomal preparations of amygdala fractions. Synaptosomes from each mouse were incubated overnight at room temperature with endoneuraminidase-N (AbCys, Paris, France; final dilution 1:120) to selectively cleave the PSA moiety of NCAM. The reaction was stopped by boiling samples at $100^{\circ} \mathrm{C}$ for $5 \mathrm{~min}$ in $70 \mathrm{mM}$ Tris- $\mathrm{HCl}$ (pH 6.8), $33 \mathrm{mM} \mathrm{NaCl}, 1 \mathrm{mM}$ EDTA, $2 \%$ sodium dodecyl sulphate (SDS), $0.01 \%$ bromophenol blue, $10 \%$ glycerol and $3 \%$ dithiothreitol. About $3.5 \mu \mathrm{g}$ of total protein from each sample was separated on $7.5 \%$ sodium dodecyl sulphate-polyacrylamide gel electrophoresis (SDS-PAGE) and transferred to a nitrocellulose membrane (Protran, Whatman $\mathrm{GmbH}$, Dassel, Germany). After saturation of non-specific sites with $5 \%$ nonfat dry milk in $10 \mathrm{mM}$ Tris $-\mathrm{HCl}(\mathrm{pH} 7.4)$, containing $150 \mathrm{mM} \mathrm{NaCl}, 0.05 \%$ Tween-20 (TBST), the membranes were probed for $2 \mathrm{~h}$ at room temperature with primary antibodies against NCAM (1:5000, Millipore, Temecula, CA, USA) or actin (1:20,000, Sigma, St. Louis, MO, USA), washed with TBST, incubated for $2 \mathrm{~h}$ with the appropriate secondary horseradish peroxidase-linked antibodies, and finally developed using the SuperSignal West Dura Substrate (Pierce, Rockford, IL, USA). Bands were detected using the ChemiDoc XRS system (Biorad, Hercules, CA, USA) and densitometry analysis on band was calculated using Biorad Quantity One 4.2.3 software (Biorad Laboratories AG, Switzerland). Following normalization to within-lane actin (data not shown), the expression 
of all three main NCAM isoforms was expressed as the percentage of the control animals. The linear range of specific antibody signal detection was determined at the outset of these experiments and all experimental samples were loaded at a concentration within the linear range of the antibody signal detection.

\section{ELISA for PSA-NCAM assessment}

PSA-NCAM levels were quantified in the same samples that were used for NCAM quantification by performing commercial PSANCAM ELISAs (AbCys Paris, France). A total volume of $100 \mu \mathrm{l}$ of each sample was loaded at a concentration of $4 \mu \mathrm{g} / \mathrm{ml}$ per well in duplicates and amounts of PSA were estimated according to the manufacturer's protocol. PSA-NCAM levels (ng PSA/ $\mu$ g of total protein) were calculated and normalized to total NCAM expression.

\section{Corticosterone Assay}

Basal corticosterone levels were measured in chronically stressed and control C57BL/6J wild-type mice ( $n=8-9$ /group), as previously described (Bisaz et al., 2009b). Briefly, mice were decapitated on the morning of day 29 (24-h after the last applied stressor) and a sample of trunk blood was collected. The blood was centrifuged (2000 $\boldsymbol{g}$ for $5 \mathrm{~min}$ and $4^{\circ} \mathrm{C}$ ) and the serum was extracted and stored at $-20^{\circ} \mathrm{C}$ until the corticosterone levels were assayed by ELISA (Assay Design, Ann Arbor, MI, USA).

\section{Auditory fear conditioning}

Training and testing took place in a rodent observation cage $(20 \times 20$ $\times 28 \mathrm{~cm}$ ), placed into a sound-attenuating chamber, illuminated by a 20-W bulb. The side walls of the observation cage were constructed of white methacrylate, the door and the top cover of Plexiglass. The floor consisted of 20steel rods through which a scrambled shock from a shock generator could be delivered. Ventilation fans provided a background noise of $68 \mathrm{~dB}$ (whole system: Panlab, S.L., Barcelona, Spain).

Fear conditioning to a tone was performed 2 days after cessation of the chronic stress procedure in chronically stressed and control mice ( $n=7-11 /$ group). Additionally auditory fear conditioning was conducted in age-matched conditional NCAM-deficient mice (NCAMffcre) and their control (NCAMff) littermates ( $n=7-8 /$ group). On the day of acoustic fear conditioning, mice were transported from the colony room to the adjacent behavioral laboratory and placed in the conditioning chamber. In the auditory fear conditioning sessions, mice were exposed to Context A (white rectangular walls, steel grid floor, white lights, cleaned with $1 \%$ acetic acid before each trial) during 160 seconds, followed by three presentations of tone-shock pairings in which the tone $(20$ seconds, $80 \mathrm{~dB}, 1000 \mathrm{~Hz}$ ) co-terminated with a foot shock (0.4 mA, 2 seconds). The intertone interval was 40 seconds and the conditioning session lasted 360 seconds in total. Twenty-four hour following auditory fear conditioning training mice were put into Context B (green round walls, gray plastic floor, cleaned with vanilla flavored water before each trial) for $8 \mathrm{~min}$ in total and confronted with the same tone as in training during the last $5 \mathrm{~min}$. After training and testing sessions, animals were immediately returned to their home cages. Animals' behavior was recorded and later scored with in-house-made behavior observation software by an observer blind to the genotype and treatment of the animals. Indicator of fear memory was freezing, defined as a lack of all movements except for respiratory-related movements. Freezing time was transformed to percentage freezing levels.

\section{Dark-light box test}

Anxiety-related behaviors were evaluated in the light-dark box 1 day after chronic stress exposure in chronically stressed and control C57BL/6J wild-type mice ( $n=8-11 /$ group) and in naïve conditional NCAM-deficient mice ( $n=11-14 /$ group). Briefly, mice were placed in the center of the dark compartment $(27 \times 27 \times 26 \mathrm{~cm})$, which was covered by a lid. The test was run for $10 \mathrm{~min}$. The adjacent white light compartment $(27 \times 27 \times 26 \mathrm{~cm})$ was connected by an opening and was illuminated from above with 45 Lux. Total number of exits, time spent in the light compartment, latency to first exit and average velocity in the light compartment were recorded by a video tracking system (Ethovision 3.0, Noldus, Wageningen, Netherlands)

\section{Open field test}

Because of technical limitations, anxiety-related behaviors of chronically stressed and control conditional NCAM-deficient mice ( $n=6 /$ group) were assessed in the open field (OF) test as previously described (Jakobsson et al. 2008; Markram et al. 2007a), instead of in the light-dark box as above. Briefly, mice were placed in the center of a white quadratic box $(50 \times 50 \times 37 \mathrm{~cm})$ and were allowed to move freely during $10 \mathrm{~min}$, while their trajectories were recorded by a video tracking system (Ethovision 3.0 Noldus, Wageningen, Netherlands). Total number of entries and time spent in the center area (25 $\times 25 \mathrm{~cm}$ virtual square in the middle of the OF) as well as average distance to the center point (center of gravity of the arena) served as indicators of anxiety-related behaviors, while total distance and average velocity were used as an index of locomotor activity.

\section{Data analysis}

All the results were expressed as the mean \pm standard error of the mean (SEM) and they were analyzed with StatView Version 5 (SAS Institute, Cray, NC). The data were analyzed with unpaired Student's t-tests or repeated measures analysis of variance (ANOVA). Post-hoc tests (PLSD Fisher) were applied when ANOVAs yielded significant interactions. Significance of the results was accepted at $P \leq 0.05$.

\section{Results}

\section{Effect of chronic unpredictable stress on body weight and basal plasma corticosterone}

The stressful nature of the chronic unpredictable stress protocol was verified by measuring body weight changes and basal plasma corticosterone levels in chronically stressed and control C57BL/6J wild-type mice. Stressed animals showed a significant body weight loss after 28 days of chronic unpredictable stress exposure $(-2.2 \pm 0.3 \mathrm{~g}, n=$ 17), whereas control mice significantly gained weight during the same time period $(0.7 \pm 0.1 \mathrm{~g}, n=19) \quad\left(t_{34}=8.391\right.$, $P<0.0001$; data not shown).

Trunk blood samples were taken on the morning of day 29 to measure basal corticosterone levels. Chronically stressed animals displayed a tendency toward higher basal plasma corticosterone levels $(58.3 \pm 9.7 \mathrm{ng} / \mathrm{ml}, n=9)$ when compared with control mice $(40.2 \pm 5.2 \mathrm{ng} / \mathrm{ml}, n=8)$, which approached statistical significance $\left(t_{15}=-1.579, P=0.07\right)$. This tendency is in agreement with a broad body of data showing similar results in mice and rats submitted to chronic stress (Bisaz et al. 2009b; Li et al. 2008; Sandi \& Touyarot 2006; Touyarot \& Sandi 2002; Venero et al. 2002).

\section{NCAM and PSA-NCAM expression in the amygdala of chronically stressed C57BL/6J wild-type mice}

Repeated measures ANOVA showed a trend toward significance in the 'stress' factor on the overall expression of the three major NCAM isoforms (Fig. 1a; $F_{1,15}=3.352$ $P=0.08)$ but no significance in the 'stress $\mathrm{x}$ isoform' interaction $\left(F_{2,30}=0.490, P>0.1\right)$. Student's $t$-tests further showed significant effects of stress on the expression of 
a

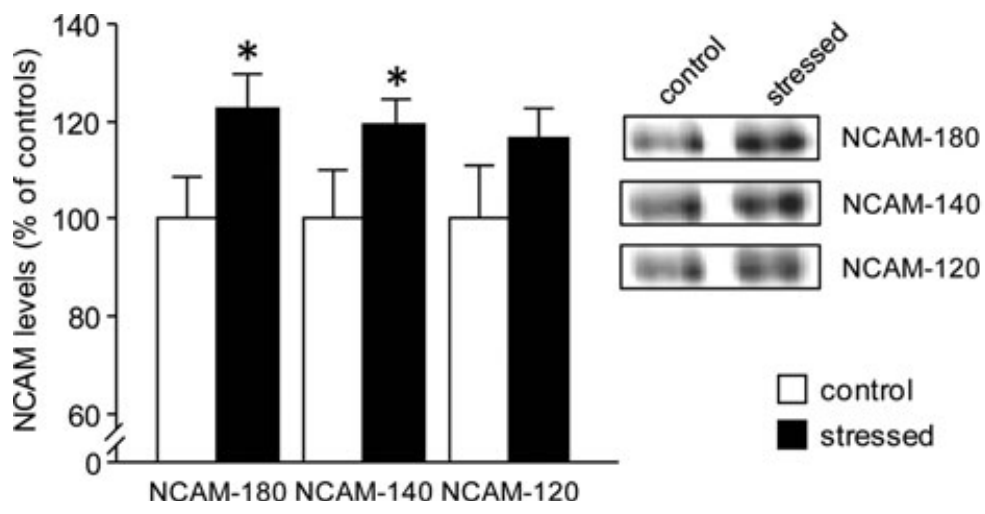

b

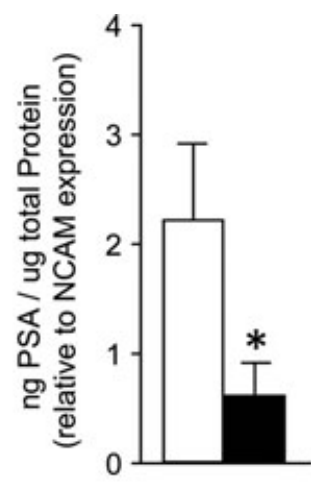

Figure 1: NCAM and PSA-NCAM expression levels in the amygdala of chronically stressed C57BL/6J wild-type mice. (a) Stressed mice displayed a significant increased of the NCAM-180 and NCAM-140 isoforms in the amygdala when compared with controls. (b) PSA-NCAM levels in relation to NCAM expression were decreased in the amygdala of stressed mice when compared with controls. Results are the mean \pm SEM ( $n=8-9 /$ group; ${ }^{*} P<0.05$ versus controls).

the two predominantly neuronal NCAM isoforms, NCAM$180\left(t_{15}=-2.071, P<0.03\right)$ and NCAM-140 $\left(t_{15}=-1.808\right.$, $P<0.05)$, with higher expression levels detected in stressed than in control animals (Fig. 1a). No significant difference was detected for the predominantly glial-expressed NCAM120 isoform ( $\left.t_{15}=-1.385, P=0.1\right)$. In addition, chronically stressed mice displayed an overall decrease of PSA-NCAM levels in the amygdala when compared with controls (Fig. 1b; $\left.t_{15}=4.707, P<0.05\right)$.

\section{Effect of chronic stress on auditory fear conditioning in C57BL/6J wild-type mice}

As shown in Fig. 2, repeated measures ANOVA for freezing levels during the postshock period (i.e., during and following tone delivery) at training showed a significant effect for the 'stress' factor $\left(F_{1,16}=5.557, P<0.05\right)$, with higher freezing levels observed in chronically stressed mice $(n=7)$, when compared with control mice $(n=11)$. However, ANOVAs indicated a lack of effect for the 'time' factor $\left(F_{2,32}=1.904\right.$, $P>0.1)$ or for the 'time $\mathrm{x}$ stress' interaction $\left(F_{2,32}=0.872\right.$, $P>0.1)$.

During tone presentation in the 24-h test, repeated measures ANOVA revealed that stressed animals showed significantly higher freezing levels than controls $\left(F_{1,16}=4.807\right.$, $P<0.05)$. No significant effect was found for the 'time' factor $\left(F_{4,64}=0.693, P>0.1\right)$ or for the 'time $\mathrm{x}$ stress' interaction $\left(F_{4,64}=2.136, P=0.09\right)$. It should be noted that stressed mice displayed higher freezing levels during the pretone session of the 24 -h test $(79.1 \pm 4.3 \%)$, when compared with controls $\left(57.7 \pm 4.4 \% ; F_{1,16}=5.012, P<0.05\right)$.

\section{Anxiety-like behaviors of chronically stressed C57BL/6J wild-type mice in the dark/light box}

We then tested anxiety-like behavior in the dark-light box test. A new set of C57BL/6J wild-type male mice was either chronically stressed for 28 days $(n=8)$ or left undisturbed

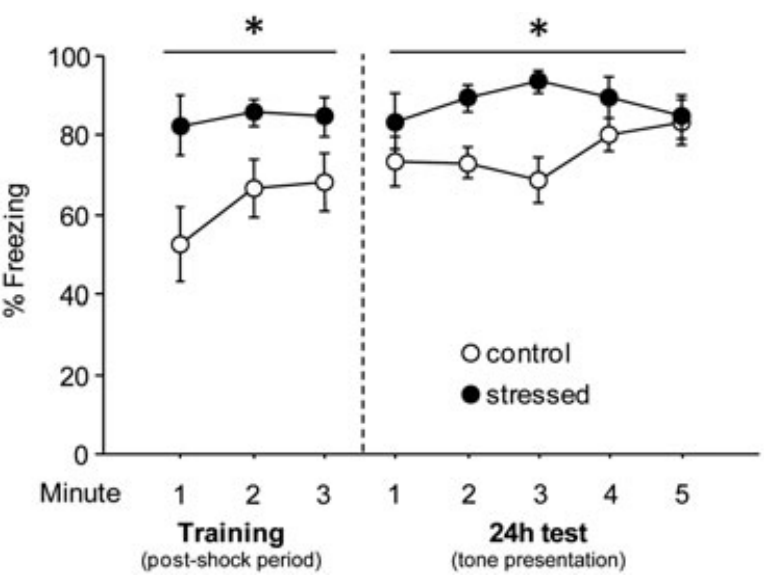

Figure 2: Freezing response of chronically stressed and control C57BL/6J wild-type mice during auditory fear conditioning. Stressed mice displayed signifcant higher freezing levels during the postshock period at training as well as during the tone presentation in the 24-h test when compared with controls. Results are the mean \pm SEM ( $n=7-11 /$ group; ${ }^{*} P<0.05$ versus controls).

( $n=11$ ) before being tested in the dark-light box test. Stressed mice displayed a significantly reduced total number of exits (Fig. 3a; $t_{17}=2.278, P<0.05$ ), reduced time spent in (Fig. $3 b ; t_{17}=1.807, P<0.05$ ) and higher latency to enter (Fig. 3c; $t_{17}=-3.156, P<0.01$ ) the light compartment than controls. These behavioral changes are conventionally interpreted as indication of higher anxiety. No difference in the average velocity in the light compartment was found between stressed and control mice (Fig. $3 d ; t_{17}=0.264$, $P>0.1$ ), indicating no general alterations of broad locomotor behavior by chronic stress. 
a

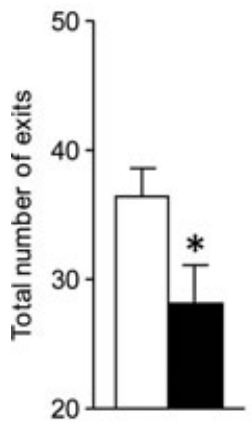

b

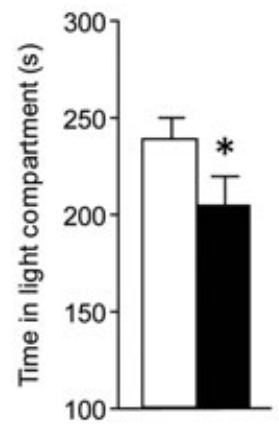

C

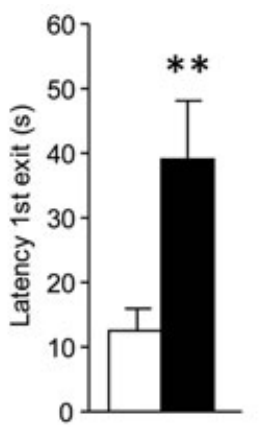

d

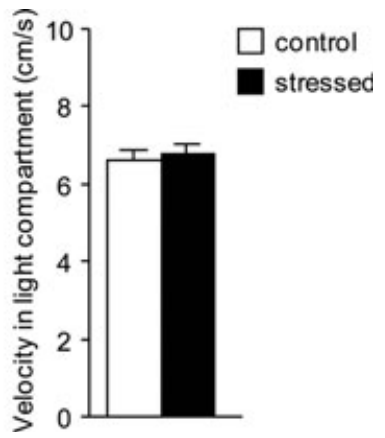

Figure 3: Anxiety-like behaviors in the dark-light box test of chronically stressed C57BL/6J wild-type mice. Chronically stressed mice vistied less frequent (a), spent less time (b) and displayed a higher latency (c) to enter the light compartment when compared with control mice. (d) No difference in the average velocity in the light compartment was found between stressed and control mice. Results are the mean \pm SEM ( $n=8-11 /$ group; ${ }^{*} P<0.05$ versus controls; ${ }^{*} P<0.01$ versus controls).

\section{NCAM and PSA-NCAM expression in the amygdala of conditional NCAM-deficient mice}

NCAM expression levels of conditional NCAM-deficient (NCAMffcre) mice were evaluated in crude synaptosomal preparations of total amygdala fraction obtained from naïve NCAMffcre mice $(n=6)$ and their NCAMff littermates $(n=4)$. Repeated measures ANOVAs indicated a significant effect of the genotype $\left(F_{1,8}=17.724, P<0.01\right)$ on the overall NCAM expression levels for all the three major NCAM isoforms, with NCAMffcre mice showing significantly less NCAM in the amygdala than their NCAMff littermates (Fig. 4a). ANOVA indicated that there was no significant 'genotype $\times$ NCAM isoform' interaction $\left(F_{2,16}=1.934\right.$, $P>0.1$ ). Moreover, Student's $t$-tests for each NCAM isoform showed reduced levels of each NCAM isoform in NCAMffcre animals when compared with NCAMff littermates (Fig. 4a; NCAM-180, $t_{8}=5.590, \quad P<0.001$; NCAM-140, $t_{8}=3.332, P<0.05 ;$ NCAM-120, $t_{8}=3.151$, $P<0.05)$. No differences in PSA-NCAM expression relative to NCAM levels were found in the amygdala between the two genotypes (Fig. 4b; $t_{8}=0.054, P>0.1$ ), indicating that the relative polysialylation of NCAM molecules is not altered in conditional NCAM-deficient mice.

\section{Auditory fear conditioning of conditional NCAM-deficient mice}

Auditory fear conditioning was tested in naïve NCAMffcre mice $(n=7)$ and their NCAMff littermates $(n=9)$ following the same protocol used for the chronic stress experiment. a

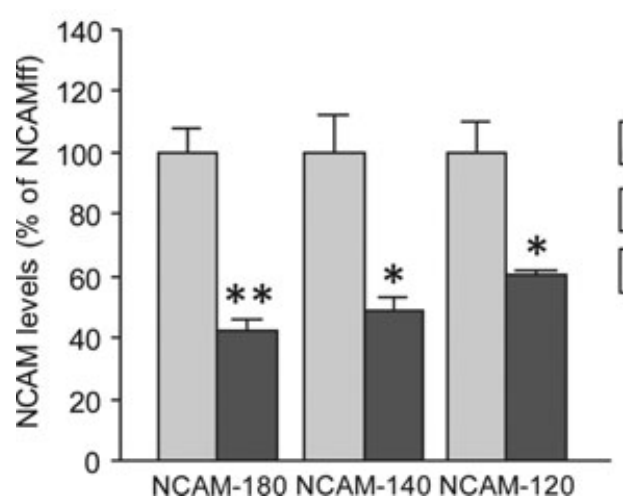

b

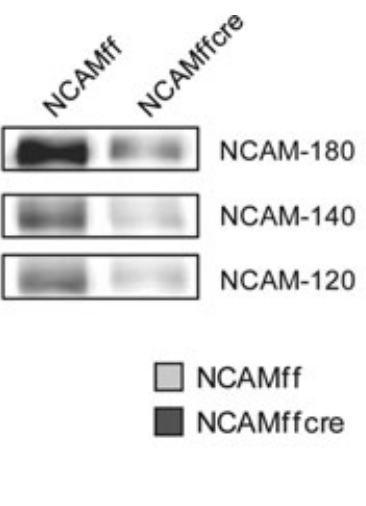

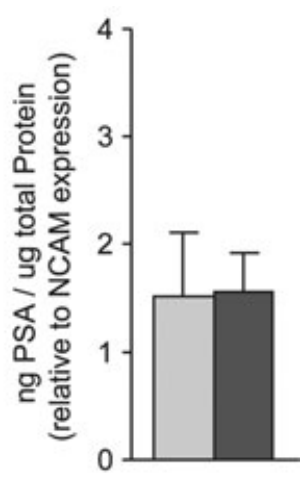

Figure 4: NCAM and PSA-NCAM expression levels in the amygdala of conditional NCAM-deficient mice. (a) Conditional NCAMdeficient mice (NCAMffcre) express sigificantly less of all major NCAM isoforms in the amygdala when compared with their NCAMff littermates. (b) No difference in PSA-NCAM in relation to NCAM expression was found in the amygdala of NCAMffcre mice when compared with NCAMff littermates. Results are the mean \pm SEM $\left(n=4-6 /\right.$ group; ${ }^{*} P<0.05$ versus NCAMff; ${ }^{*} P<0.01$ versus NCAMff). 
As shown in Fig. 5, repeated measures ANOVA on data from the postshock period showed no significant effect for the 'genotype' factor $\left(F_{1,14}=1.273, P>0.1\right)$, indicating similar freezing levels of animals from both genotypes. In addition, ANOVAS also revealed a significant effect for the 'time' factor $\left(F_{2,28}=9.917, P=0.0006\right)$ but a lack of effect for the 'time $\times$ genotype' interaction $\left(F_{2,28}=0.216, P>0.1\right)$. At the 24-h test, NCAMffcre mice displayed significantly lower freezing levels than NCAMff littermates $\left(F_{1,14}=5.509\right.$, $P<0.05)$, while no differences were observed for the 'time' factor $\left(F_{4,56}=0.926, P>0.1\right)$ of for the factors' interaction $\left(F_{4,56}=1.842, P>0.1\right)$. In addition, mice of both genotypes showed comparable freezing levels during the pretone session of the 24-h test (NCAMffcre: $53.0 \pm 5.5 \%$; NCAMff: $\left.58.7 \pm 3.5 \% ; F_{1.14}=0.461, P>0.1\right)$.

\section{Anxiety-like behaviors of conditional NCAM-deficient mice in the dark/light box}

Anxiety-like behaviors were tested in naïve adult NCAMffcre mice $(n=11)$ and their NCAMff littermates $(n=14)$ following the same protocol than the one used for the chronic stress experiment in wild-type mice. As shown in Fig.6, NCAMffcre mice displayed significantly higher total number of exits (Fig. $6 a ; t_{23}=2.528, P<0.01$ ) and spent significantly longer time in the light compartment (Fig. $6 b ; t_{23}=2.592, P<0.01$ ) than NCAMff littermates. No significant differences were found in the latency to enter (Fig. $6 c ; t_{23}=0.554, P>0.1$ ) or on the average velocity in the light compartment (Fig. $6 \mathrm{~d} ; t_{23}=0.577, P>0.1$ ). These behavioral alterations are conventionally interpreted as indicative of diminished anxiety-like levels.

\section{NCAM and PSA-NCAM expression in the amygdala of chronically stressed conditional NCAM-deficient mice}

NCAM expression levels were further evaluated in the amygdala and hippocampus of chronically stressed $(n=5)$ and

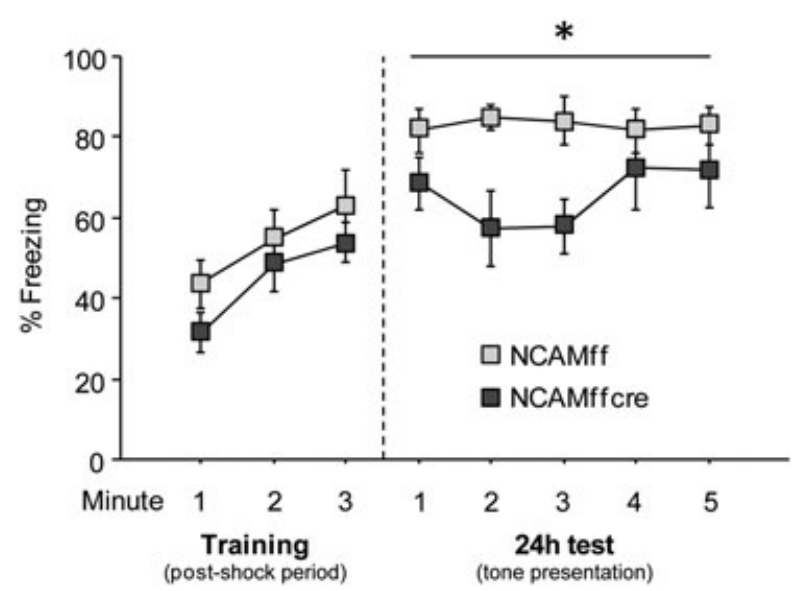

Figure 5: Freezing response of conditional NCAM-deficient mice during auditory fear conditioning. No difference in freezing levels was found between conditional NCAM-deficient mice (NCAMffcre) and their NCAMff littermates during the postshock period at training. However, NCAMffcre mice displayed significant lower freezing levels during the tone presentation of the 24-h tone test when compared with NCAMff mice. Results are the mean \pm SEM $\left(n=7-9 /\right.$ group; ${ }^{*} P<0.05$ versus NCAMff).

control $(n=5)$ conditional NCAMffcre mice. In amygdala crude synaptosomal preparations (Fig. 7a), repeated measures ANOVA for the three major NCAM isoforms showed no significant overall effect of stress (Fig. 7a; $F_{1,8}=0.021$, $P>0.1)$ as well as no significant 'NCAM isoform $\times$ stress' interaction $\left(F_{2,16}=0.525, P>0.1\right)$. Student's $t$-tests for each isoform further showed no significant effects of stress (NCAM-180: $t_{8}=-0.365, P>0.1 ;$ NCAM-140: $t_{8}=0.087$, $P>0.1 ;$ NCAM-120: $\left.t_{8}=0.374, P>0.1\right)$. As shown in Fig. S1, repeated measures ANOVA for the three major NCAM isoforms in hippocampal crude synaptosomal preparations a

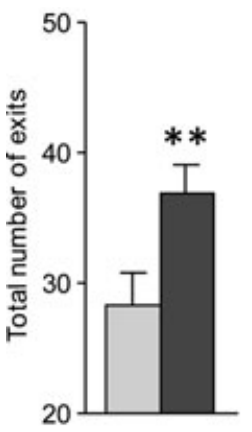

b

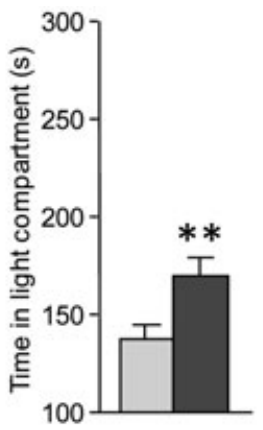

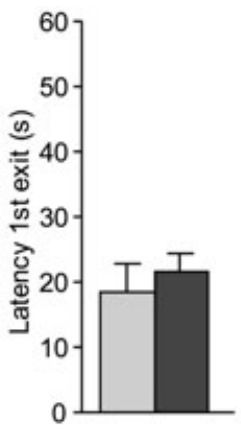

d

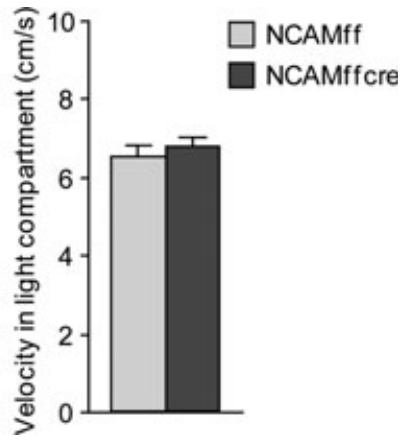

Figure 6: Anxiety-like behaviors in the dark-light box test of conditional NCAM-deficient mice. Conditional NCAM-deficient mice (NCAMffcre) visited more frequent (a) and spent more time (b) in the light compartment when compared with NCAMff littermates. No difference in the latency to enter (c) and the average velocity (d) in the light compartment was found between NCAMffcre and NCAMff littermates. Results are the mean $\pm \operatorname{SEM}\left(n=11-14 /\right.$ group; ${ }^{*} P<0.01$ versus NCAMff). 
a

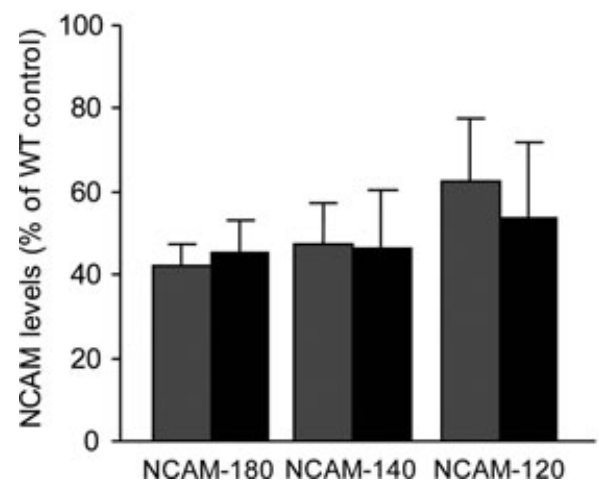

b

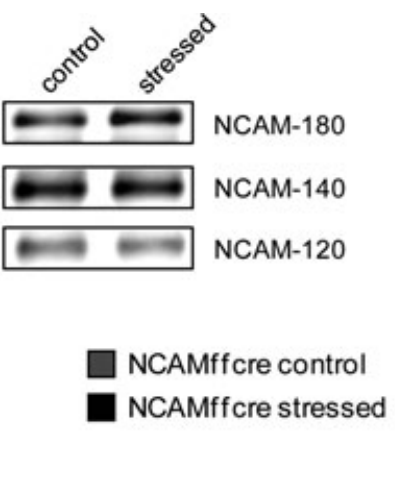

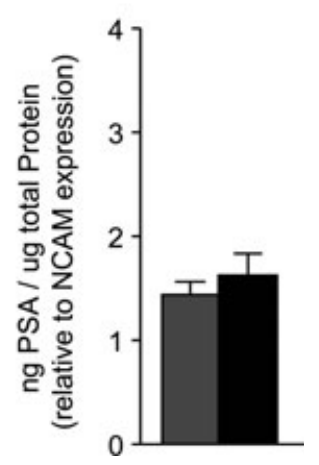

Figure 7: NCAM and PSA-NCAM expression levels in the amygdala of chronically stressed conditional NCAM-deficient mice. No signifcant differences in NCAM (a) and PSA-NCAM (b) expression levels were found in the amygdala between stressed and control conditional NCAM-deficient (NCAMffcre) mice. Results are the mean $\pm \operatorname{SEM}$ ( $n=5 /$ group).

showed no significance effects, neither for the 'stress' factor $\left(F_{1,8}=0.129, P>0.1\right)$ nor for the 'NCAM isoform $\times$ stress' interaction $\left(F_{2,16}=0.284, P>0.1\right)$. Similarly, Student's $t$-test showed no significant effect of stress on the expression of any of the three major NCAM isoforms (NCAM-180: $t_{8}=0.131, P>0.1 ; \mathrm{NCAM}-140 ; t_{8}=0.264, P>0.1 ; \mathrm{NCAM}-$ 120: $t_{8}=0.857, P>0.1$ ). These data indicate that, in conditional NCAM-deficient mice, NCAM expression levels in the amygdala and hippocampus are not altered by chronic stress. No difference in PSA-NCAM levels relative to NCAM were found between chronically stressed and control NCAMffcre mice, neither in the amygdala (Fig. 7b; $t_{8}=-0.863, P>0.1$ ), nor in the hippocampus (Fig. S1; $t_{8}=-1.237, P>0.1$ ) indicating that relative polysialylation of NCAM molecules in these brain structures was not altered by stress in conditional NCAM-deficient mice.

\section{Auditory fear conditioning of chronically stressed conditional NCAM-deficient mice}

We then tested auditory fear conditioning in chronically stressed $(n=6)$ and control $(n=6)$ NCAMffcre mice following the same protocol as previously used. As shown in Fig. 8, repeated measures ANOVA on data from the postshock period at training showed a significant difference in the freezing levels between stressed and control NCAMffcre mice $\left(F_{1,10}=11.299, \quad P<0.01\right)$. ANOVA also showed a significant effect for the 'time' factor $\left(F_{2,20}=3.783, P<\right.$ 0.05 ) but a lack of effect for the 'time $\times$ stress' interaction $\left(F_{2,20}=0.654, P>0.1\right)$. As to the repeated measures ANOVA on data from the 24-h test, stressed and control NCAMffcre mice showed comparable freezing levels (Fig. $7 ; F_{1,10}=$ $0.846, P>0.1)$. No significant effect was observed for the 'time' factor $\left(F_{4,40}=0.989, P>0.1\right)$ or for the 'time $\times$ stress' interaction $\left(F_{4,40}=0.158, P>0.1\right)$. Similar to C57BL/6J wildtype mice, stressed NCAMffcre mice displayed significantly higher freezing levels during the pretone period at the 24-h test $(80.7 \pm 5.1 \%)$ than control NCAMffcre animals $(50.4 \pm$ $\left.7.0 \% ; F_{1,10}=5.269, P<0.05\right)$.

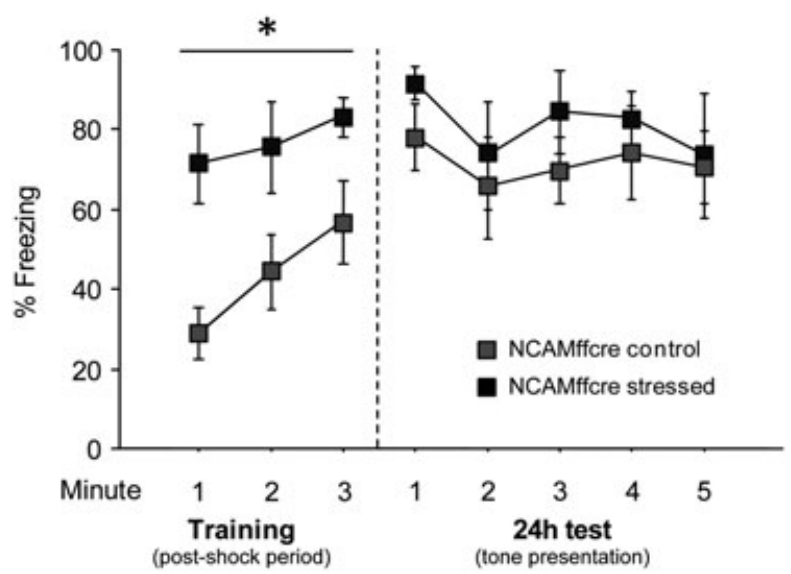

Figure 8: Freezing response of chronically stressed and control conditional NCAM-deficient mice during auditory fear conditioning. Stressed conditional NCAM-deficient mice (NCAMffcre) displayed significant higher freezing levels during the postshock period at training. However, no significant difference in the freezing levels during the tone presentation in the 24-h tone test was found between stressed and control NCAMffcre mice. Results are the mean \pm SEM ( $n=6 /$ group; ${ }^{*} P<0.05$ versus NCAMff).

\section{Anxiety-like behaviors of chronically stressed conditional NCAM-deficient mice in the open field test}

Because of technical limitations, anxiety-like behaviors in chronically stressed conditional NCAM-deficient (NCAMffcre) mice were examined in the open field (OF) test instead of in the dark-light box. Stressed $(n=6)$ and control $(n=6)$ NCAMffcre were tested 1 day after the cessation of the chronic stress procedure. The OF test relies on the use of an open area with an exposed and 'anxiogenic' center and a less exposed periphery. In this test, the natural 


\section{Bisaz and Sandi}

tendency of animals to explore the arena competes with their tendency to avoid the open, central area. Chronically stressed NCAMffcre mice displayed a reduced total number of entries $\left(t_{10}=2.142, P<0.05\right)$ and spent less time $\left(t_{10}=2.591, P<0.05\right)$ in the center of the OF than control NCAMffcre mice (see Table S1). In addition, stressed NCAMffcre mice showed a significantly higher average distance to the center point of the OF during the 10-min test $\left(t_{10}=-3.137, P<0.01\right)$ than control NCAMffcre mice. These measurements are indicative of higher anxiety-levels in stressed NCAMffcre animals. No significant differences between stressed and control NCAMffcre mice were found neither in the total distance $\left(t_{10}=1.358, P>0.1\right)$, nor in the average velocity $\left(t_{10}=1.358, P>0.1\right)$, indicating no general alterations of broad locomotor behavior induced by chronic stress in these mutant mice.

\section{Discussion}

We show here that a chronic unpredictable stress protocol applied in C57BL/6 J wild-type mice during 28 days, enhances anxiety-like behavior and auditory-cued fear conditioning, and increases NCAM levels in the amygdala. We also show that mice, in which the NCAM gene is ablated in glutamatergic neurons of the forebrain by the second postnatal week (Bukalo et al. 2004), display low levels of NCAM in the amygdala which are accompanied by reduced anxiety and auditory fear conditioning. Moreover, we report that, if chronically stressed, conditional NCAM-deficient mice do not show changes in auditory fear conditioning or in NCAM and PSA-NCAM expression levels in the amygdala (nor in the hippocampus), while showing evidence for increased anxiety-like behaviors. These findings suggest a role for NCAM in the amygdala in auditory fear conditioning and for stress-increased amygdala NCAM expression in the mechanisms whereby stress potentiates fear conditioning.

Chronic stress potentiates amygdala-dependent behaviors, as shown previously in rats for anxiety-like behavior and fear conditioning (Conrad et al. 1999; Cordero et al. 2003; Sandi et al. 2001), and confirmed in this study in mice. Moreover, we found that chronically stressed mice displayed increased fear (i.e. freezing) upon shock delivery at training. This observation is in agreement with the wellcharacterized phenomenon in rats, previously submitted to inescapable footshocks, of enhanced freezing when exposed to footshocks in a new environment (Maier 1990; Rau et al. 2005), an effect that was related to learned helplessness and behavioral sensitization (MacLennan et al. 1982; Maier et al. 1993; Rau et al. 2005). Strikingly, we found here that the impact of chronic stress on NCAM expression in the amygdala is the opposite to the reduced expression found in the hippocampus (Alfonso et al. 2006; Bisaz et al. 2009b; Sandi et al. 2001; Sandi \& Touyarot 2006; Touyarot \& Sandi 2002; Touyarot et al. 2004; Venero et al. 2002): stress increased the two mainly neuronal-expressed isoforms NCAM-180 and NCAM-140, without significantly affecting the predominately glial-expressed splice variant NCAM-120.

A main question that arises from these findings is whether there is a link between the stress-induced increase in
NCAM expression in the amygdala and amygdala-related functional alterations. In this study, we specifically addressed the connection with amygdala-related behaviors by using conditional NCAM-deficient mice in which NCAM is ablated under the control of the $\alpha$ CaMKII promoter predominantly in hippocampal neurons postnatally (Bukalo et al. 2004). First, we verified that this mutation leads to reduced expression of NCAM in the amygdala and found consistent effects in the three main isoforms. Second, we questioned whether this pattern of NCAM expression in the amygdala of conditional NCAM-deficient mice - which is the reverse to changes observed in chronically stressed mice - would be related to alterations in amygdala-related behaviors opposite to those found after chronic stress. As hypothesized, we show that NCAM-deficient mice display reduced anxiety-like behavior (as assessed in the light-dark box) and reduced auditory fear conditioning. Although the alteration in anxiety-like behavior differs from the increased levels described in constitutive NCAM knockout mice (Cremer et al. 1994; Stork et al. 1997; Stork et al. 1999), the deficit in auditory fear conditioning is in agreement with similar changes reported in constitutive NCAM knockout mice (Senkov et al. 2006; Stork et al. 2000). Altogether, these results suggest a role for NCAM in the amygdala in auditory fear memories, but question the link between this molecule and anxiety-like behavior. However, it should be noted that constitutive NCAM knockout mice present important morphological alterations because of the absence of NCAM and to the complete lack of both NCAM and its posttranslational modification - addition of long chains of polysialic acid to NCAM (PSA-NCAM) - during development (Chazal et al. 2000; Cremer et al. 1997). On the contrary, no overt morphological alterations were found in the forebrain of the conditional NCAM mutant mice used in this study (Bukalo et al. 2004) and, therefore, they can be considered as a more adequate model to study the functional consequences of reduced NCAM expression than the constitutive mutants. Moreover, the fact that NCAM levels in the amygdala are reduced, but not completely abolished, and that the ratio of PSA-to-NCAM is not altered, makes it a particularly suitable model to establish links between changes in NCAM expression and behavior.

To causally relate NCAM to stress-induced changes in amygdala-related behaviors, we submitted conditional NCAM-deficient mice to chronic stress and tested them in the auditory fear conditioning task and assessed anxietylike behaviors in the OF. We reasoned that if the observed stress-induced increases in amygdala NCAM expression would be involved in the coincident enhancement of auditory fear conditioning and/or anxiety-like behavior, submitting NCAM-deficient mice to chronic stress should not affect, respectively, their conditioned fear and anxietylike responses. Our results agreed with this prediction for fear conditioning, further supporting a possible role for NCAM in stress-induced alterations in amygdala function and, more specifically, in enhanced auditory-cued fear conditioning. It should be noted that these behavioral alterations in chronically stressed conditional NCAM-deficient mice occurred without affecting NCAM expression levels neither in the amygdala nor in the hippocampus. This experiment also showed increased freezing in the stressed 
conditional NCAM-deficient mice as an immediate reaction to the shock received at training, as compared with unstressed mice from the same genotype. This increase mirrors the one observed in chronically stressed wild-type mice and differs from the lack of effect of the conditional NCAM mutation by itself on this behavioral variable, suggesting that NCAM is not involved in these 'innate' fear reaction (as opposed to the 'learned' fear conditioning). Importantly, we also found that chronic stress increased anxiety-like behaviors (as evaluated in the OF test) in conditional NCAM-deficient mice. These results, together with the inconsistency in the anxiety phenotype of constitutive and conditional NCAM mutant mice, seem to rule out a role for NCAM in innate fear reactions and anxiety, while supporting a role in the consolidation of fear conditioning and in stress-induced potentiation of fear conditioning. In fact, a wide body of evidence highlights a key role for NCAM in the hippocampus in memory consolidation (for reviews see Bisaz et al. 2009a; Conboy et al. 2008) and, therefore, suggests that the increased NCAM levels in the amygdala might contribute to a facilitation of fear memory processes mediated by this brain region (Kim \& Fanselow 1992; Phillips \& Ledoux 1992).

As a note of caution, it is important to note that the conditional NCAM-deficient mice used in our study were previously shown to display reduced NCAM levels in the hippocampus and cerebral cortex, including the prefrontal cortex (Bisaz etal. 2009b; Bukalo et al. 2004), similarly mimicking the effect of chronic stress exposure (Bisaz et al. 2009b). Whereas the initial view was that auditory fear conditioning depends on an intact amygdala, but not hippocampus (Kim \& Fanselow 1992; Phillips \& Ledoux 1992), supporting an essential role of the amygdala (as opposed to the hippocampus) on this type of learning, there is mounting evidence questioning the anatomical segregation of cue and context conditioning and suggesting that the hippocampus might participate in certain types of auditory fear conditioning (Maren 2008; Quinn et al. 2008). However, our results showing opposite effects on auditory fear conditioning (this study) under conditions of reduced NCAM expression in the hippocampus induced either by chronic stress or through genetic manipulations (Bisaz et al. 2009b) do not seem to support a role for hippocampal NCAM in this type of cued conditioning.

Reduced NCAM expression has been proposed to play a key role in the structural shrinkage and learning impairments which are observed as a consequence of chronic stress in the hippocampus and prefrontal cortex (Sandi 2004; Sandi \& Bisaz 2007). Evidence in rats suggests that chronic stress might also affect dendritic branching and connectivity in the amygdala and the potential involvement of NCAM has not been previously addressed. At the structural level, chronic immobilization (but not unpredictable) stress was shown to induce, in rats, a persistent increase in dendritic branching [also apparent after glucocorticoid treatment (Mitra \& Sapolsky 2008; Vyas et al. 2002)] and in spine density (Mitra et al. 2005; Vyas et al. 2002; Vyas et al. 2004), whereas chronic corticosterone reduced dendritic arborization (Pego et al. 2008) in the BLA. Although we did not perform morphological assessments in our study and there are no data in mice targeting morphological changes induced by stress in the amygdala, given the well-characterized roles of NCAM in neuritogenesis and spine formation (for reviews see Fields \& Itoh 1996; Kiss \& Muller 2001; Muller et al. 2008; Schachner 1997), it is tempting to speculate that the stress-induced increase in NCAM levels described in this study might contribute to structural remodeling. Future studies should specifically address this important question.

As previously reported in rats (Cordero et al. 2005), we found that chronic stress reduced PSA-NCAM expression in the amygdala of chronically stressed mice. It should be noted that conditional NCAM-deficient mice also present an overall (when not expressed as the ration to NCAM) reduction in PSA-NCAM levels in the amygdala. Although we did not focus here on the role of PSA-NCAM in the amygdala, previous studies failed to implicate this molecule in auditory fear conditioning (Markram et al. 2007a,b; Senkov et al. 2006). In fact, the scarce information available in the literature, suggesting a potential role for PSA-NCAM in the amygdala among the mechanisms underlying different emotional states, it is currently confusing as to its precise role. Chronic antidepressant treatment was found to reduce PSA-NCAM and synaptophysin expression in the amygdala (Varea et al. 2007), whereas exposure to stress during the juvenile period in rats - a treatment that leads to behavioral inhibition in adulthood - prevented the reduction of PSA-NCAM to NCAM ratio that occurs on the transition from adolescence to adulthood (Tsoory et al. 2008). Future work should specifically address the potential link between PSA-NCAM in the amygdala and structural and functional changes induced by stress.

In summary, the combination of chronic stress, biochemical, behavioral and genetic approaches followed in this study allowed us to present strong correlational, and partially causal, evidence highlighting, for the first time, a role for NCAM in the amygdala in auditory fear conditioning and for increased NCAM expression in the amygdala among the mechanisms whereby stress facilitates cued fear conditioning processes. Although they open new venues for potential interventions in stress-related psychopathologies, future interventive studies, including, for example, treatment with NCAM mimetic peptides, should be addressed to further verify this conclusion.

\section{References}

Alfonso, J., Frick, L.R., Silberman, D.M., Palumbo, M.L., Genaro, A.M. \& Frasch, A.C. (2006) Regulation of hippocampal gene expression is conserved in two species subjected to different stressors and antidepressant treatments. Biol Psychiatry 59, 244-251.

Bisaz, R., Conboy, L. \& Sandi, C. (2009a) Learning under stress: a role for the neural cell adhesion molecule NCAM. Neurobiol Learn Mem 91, 333-342.

Bisaz, R., Schachner, M. \& Sandi, C. (2009b) Causal evidence for the involvement of the neural cell adhesion molecule, NCAM, in chronic stress-induced cognitive impairments. Hippocampus, in press. DOI: 10.1002/hipo.20723.

Bukalo, O., Fentrop, N., Lee, A.Y., Salmen, B., Law, J.W., Wotjak, C.T., Schweizer, M., Dityatev, A. \& Schachner, M. (2004) Conditional ablation of the neural cell adhesion molecule reduces precision of spatial learning, long-term potentiation, and depression in the CA1 subfield of mouse hippocampus. J Neurosci 24, 1565-1577. 


\section{Bisaz and Sandi}

Cambon, K., Hansen, S.M., Venero, C., Herrero, A.I., Skibo, G. Berezin, V., Bock, E. \& Sandi, C. (2004) A synthetic neural cell adhesion molecule mimetic peptide promotes synaptogenesis, enhances presynaptic function, and facilitates memory consolidation. J Neurosci 24, 4197-4204.

Cambon, K., Venero, C., Berezin, V., Bock, E. \& Sandi, C. (2003) Posttraining administration of a synthetic peptide ligand of the neural cell adhesion molecule, C3d, attenuates long-term expression of contextual fear conditioning. Neuroscience 122, 183-191.

Cerqueira, J.J., Mailliet, F., Almeida, O.F., Jay, T.M. \& Sousa, N. (2007) The prefrontal cortex as a key target of the maladaptive response to stress. J Neurosci 27, 2781-2787.

Chazal, G., Durbec, P., Jankovski, A., Rougon, G. \& Cremer, H. (2000) Consequences of neural cell adhesion molecule deficiency on cell migration in the rostral migratory stream of the mouse. J Neurosci 20, 1446-1457.

Conboy, L., Bisaz, R., Markram, K. \& Sandi, C. (2008) Role of NCAM in Emotion and Learning. Neurochem Res, in press. DOI: $10.1007 /$ s11064-008-9601-4.

Conrad, C.D., Galea, L.A., Kuroda, Y. \& McEwen, B.S. (1996) Chronic stress impairs rat spatial memory on the $Y$ maze, and this effect is blocked by tianeptine pretreatment. Behav Neurosci 110, $1321-1334$

Conrad, C.D., LeDoux, J.E., Magarinos, A.M. \& McEwen, B.S. (1999) Repeated restraint stress facilitates fear conditioning independently of causing hippocampal CA3 dendritic atrophy. Behav Neurosci 113, 902-913.

Cook, S.C. \& Wellman, C.L. (2004) Chronic stress alters dendritic morphology in rat medial prefrontal cortex. Journal of Neurobiology 60, 236-248.

Cordero, M.I., Kruyt, N.D. \& Sandi, C. (2003) Modulation of contextual fear conditioning by chronic stress in rats is related to individual differences in behavioral reactivity to novelty. Brain Res 970, 242-245.

Cordero, M.I., Rodriguez, J.J., Davies, H.A., Peddie, C.J., Sandi, C. \& Stewart, M.G. (2005) Chronic restraint stress down-regulates amygdaloid expression of polysialylated neural cell adhesion molecule. Neuroscience 133, 903-910.

Cremer, H., Chazal, G., Goridis, C. \& Represa, A. (1997) NCAM is essential for axonal growth and fasciculation in the hippocampus. Mol Cell Neurosci 8, 323-335.

Cremer, H., Lange, R., Christoph, A., et al. (1994) Inactivation of the $\mathrm{N}$-CAM gene in mice results in size reduction of the olfactory bulb and deficits in spatial learning. Nature 367, 455-459.

Donohue, H.S., Gabbott, P.L., Davies, H.A., Rodriguez, J.J., Cordero, M.I., Sandi, C., Medvedev, N.I., Popov, V.I., Colyer, F.M., Peddie, C.J. \& Stewart, M.G. (2006) Chronic restraint stress induces changes in synapse morphology in stratum lacunosummoleculare CA1 rat hippocampus: a stereological and threedimensional ultrastructural study. Neuroscience 140, 597-606.

Fields, R.D. \& Itoh, K. (1996) Neural cell adhesion molecules in activity-dependent development and synaptic plasticity. Trends Neurosci 19, 473-480

Hartz, B.P. \& Ronn, L.C. (2008) NCAM in Long-Term Potentiation and Learning. Neurochem Res, in press. DOI: 10.1007/s11064008-9820-8.

Holmes, A. \& Wellman, C.L. (2009) Stress-induced prefrontal reorganization and executive dysfunction in rodents. Neurosci Biobehav Rev 33, 773-783.

Jakobsson, J., Cordero, M.I., Bisaz, R., Groner, A.C., Busskamp, V., Bensadoun, J.C., Cammas, F., Losson, R., Mansuy, I.M., Sandi, C. \& Trono, D. (2008) KAP1-mediated epigenetic repression in the forebrain modulates behavioral vulnerability to stress. Neuron 60, 818-831.

Kim, J.J. \& Fanselow, M.S. (1992) Modality-specific retrograde amnesia of fear. Science 256, 675-677.

Kiss, J.Z. \& Muller, D. (2001) Contribution of the neural cell adhesion molecule to neuronal and synaptic plasticity. Reviews in the Neurosciences 12, 297-310.
Li, S., Wang, C., Wang, M.W., Murakami, Y. \& Matsumoto, K. (2006) Impairment of the spatial learning and memory induced by learned helplessness and chronic mild stress. Pharmacology Biochemistry and Behavior 83, 186-193.

Li, S., Wang, C., Wang, W., Dong, H., Hou, P. \& Tang, Y. (2008) Chronic mild stress impairs cognition in mice: from brain homeostasis to behavior. Life Sci 82, 934-942.

Lopez-Fernandez, M.A., Montaron, M.F., Varea, E., Rougon, G., Venero, C., Abrous, D.N. \& Sandi, C. (2007) Upregulation of polysialylated neural cell adhesion molecule in the dorsal hippocampus after contextual fear conditioning is involved in long-term memory formation. J Neurosci 27, 4552-4561.

Lowry, O.H., Rosebrough, N.J., Farr, A.L. \& Randall, R.J. (1951) Protein measurement with the Folin phenol reagent. $J$ Biol Chem 193, 265-275.

Luine, V., Villegas, M., Martinez, C. \& McEwen, B.S. (1994) Repeated stress causes reversible impairments of spatial memory performance. Brain Res 639, 167-170.

Lynch, M.A. \& Voss, K.L. (1991) Presynaptic changes in long-term potentiation: elevated synaptosomal calcium concentration and basal phosphoinositide turnover in dentate gyrus. J Neurochem 56, 113-118

MacLennan, A.J., Drugan, R.C., Hyson, R.L., Maier, S.F., Madden, J.t. \& Barchas, J.D. (1982) Dissociation of long-term analgesia and the shuttle box escape deficit caused by inescapable shock. J Comp Physiol Psychol 96, 904-912.

Magarinos, A.M., Verdugo, J.M. \& McEwen, B.S. (1997) Chronic stress alters synaptic terminal structure in hippocampus. Proc Natl Acad Sci USA 94, 14002-14008.

Maier, S.F. (1990) Role of fear in mediating shuttle escape learning deficit produced by inescapable shock. J Exp Psychol Anim Behav Process 16, 137-149.

Maier, S.F., Grahn, R.E., Kalman, B.A., Sutton, L.C., Wiertelak, E.P. \& Watkins, L.R. (1993) The role of the amygdala and dorsal raphe nucleus in mediating the behavioral consequences of inescapable shock. Behav Neurosci 107, 377-388.

Maness, P.F. \& Schachner, M. (2007) Neural recognition molecules of the immunoglobulin superfamily: signaling transducers of axon guidance and neuronal migration. Nat Neurosci 10, 19-26.

Maren, S. (2008) Pavlovian fear conditioning as a behavioral assay for hippocampus and amygdala function: cautions and caveats. Eur J Neurosci 28, 1661-1666.

Markram, K., Gerardy-Schahn, R. \& Sandi, C. (2007a) Selective learning and memory impairments in mice deficient for polysialylated NCAM in adulthood. Neuroscience 144, 788-796.

Markram, K., Lopez Fernandez, M.A., Abrous, D.N. \& Sandi, C. (2007b) Amygdala upregulation of NCAM polysialylation induced by auditory fear conditioning is not required for memory formation, but plays a role in fear extinction. Neurobiol Learn Mem 87, $573-582$

Mitra, R., Jadhav, S., McEwen, B.S., Vyas, A. \& Chattarji, S. (2005) Stress duration modulates the spatiotemporal patterns of spine formation in the basolateral amygdala. Proc Natl Acad Sci USA 102, 9371-9376.

Mitra, R. \& Sapolsky, R.M. (2008) Acute corticosterone treatment is sufficient to induce anxiety and amygdaloid dendritic hypertrophy. Proc Natl Acad Sci USA 105, 5573-5578.

Muller, D., Mendez, P., De Roo, M., Klauser, P., Steen, S. \& Poglia, L. (2008) Role of NCAM in spine dynamics and synaptogenesis. Neurochem Res, in press. DOI: 10.1007/s11064-008-9653-5.

Palkovits, M. (1973) Isolated removal of hypothalamic or other brain nuclei of the rat. Brain Res 59, 449-450.

Park, C.R., Campbell, A.M. \& Diamond, D.M. (2001) Chronic psychosocial stress impairs learning and memory and increases sensitivity to yohimbine in adult rats. Biol Psychiatry 50, 994-1004.

Pavlides, C., Nivon, L.G. \& McEwen, B.S. (2002) Effects of chronic stress on hippocampal long-term potentiation. Hippocampus 12, 245-257. 
Pego, J.M., Morgado, P., Pinto, L.G., Cerqueira, J.J., Almeida, O.F. \& Sousa, N. (2008) Dissociation of the morphological correlates of stress-induced anxiety and fear. Eur J Neurosci 27, 1503-1516.

Phillips, R.G. \& LeDoux, J.E. (1992) Differential contribution of amygdala and hippocampus to cued and contextual fear conditioning. Behav Neurosci 106, 274-285.

Quinn, J.J., Wied, H.M., Ma, Q.D., Tinsley, M.R. \& Fanselow, M.S. (2008) Dorsal hippocampus involvement in delay fear conditioning depends upon the strength of the tone-footshock association. Hippocampus 18, 640-654.

Radley, J.J., Rocher, A.B., Miller, M., Janssen, W.G., Liston, C. Hof, P.R., McEwen, B.S. \& Morrison, J.H. (2006) Repeated stress induces dendritic spine loss in the rat medial prefrontal cortex. Cereb Cortex 16, 313-320.

Rau, V., DeCola, J.P. \& Fanselow, M.S. (2005) Stress-induced enhancement of fear learning: an animal model of posttraumatic stress disorder. Neurosci Biobehav Rev 29, 1207-1223.

Roozendaal, B., McEwen, B.S. \& Chattarji, S. (2009) Stress, memory and the amygdala. Nat Rev Neurosci 10, 423-433.

Sandi, C. (2004) Stress, cognitive impairment and cell adhesion molecules. Nat Rev Neurosci 5, 917-930.

Sandi, C. \& Bisaz, R. (2007) A model for the involvement of neural cell adhesion molecules in stress-related mood disorders. Neuroendocrinology 85, 158-176.

Sandi, C., Cordero, M.I., Merino, J.J., Kruyt, N.D., Regan, C.M. \& Murphy, K.J. (2004) Neurobiological and endocrine correlates of individual differences in spatial learning ability. Learn Mem 11, 244-252

Sandi, C., Davies, H.A., Cordero, M.I., Rodriguez, J.J., Popov, V.I. \& Stewart, M.G. (2003) Rapid reversal of stress induced loss of synapses in CA3 of rat hippocampus following water maze training. Eur J Neurosci 17, 2447-2456.

Sandi, C., Merino, J.J., Cordero, M.I., Touyarot, K. \& Venero, C. (2001) Effects of chronic stress on contextual fear conditioning and the hippocampal expression of the neural cell adhesion molecule, its polysialylation, and L1. Neuroscience 102, 329-339.

Sandi, C. \& Touyarot, K. (2006) Mid-life stress and cognitive deficits during early aging in rats: individual differences and hippocampal correlates. Neurobiol Aging 27, 128-140.

Schachner, M. (1997) Neural recognition molecules and synaptic plasticity. Curr Opin Cell Biol 9, 627-634.

Senkov, O., Sun, M., Weinhold, B., Gerardy-Schahn, R., Schachner, M. \& Dityatev, A. (2006) Polysialylated neural cell adhesion molecule is involved in induction of long-term potentiation and memory acquisition and consolidation in a fear-conditioning paradigm. J Neurosci 26, 10888-10898.

Shin, K.Y., Won, B.Y., Heo, C., Kim, H.J., Jang, D.P., Park, C.H., Kim, S., Kim, H.S., Kim, Y.B., Lee, H.G., Lee, S.H., Cho, Z.H. \& Suh, Y.H. (2009) BT-11 improves stress-induced memory impairments through increment of glucose utilization and total neural cell adhesion molecule levels in rat brains. J Neurosci Res 87, 260-268.

Stork, O., Welzl, H., Cremer, H. \& Schachner, M. (1997) Increased intermale aggression and neuroendocrine response in mice deficient for the neural cell adhesion molecule (NCAM). Eur $J$ Neurosci 9, 1117-1125

Stork, O., Welzl, H., Wolfer, D., Schuster, T., Mantei, N., Stork, S., Hoyer, D., Lipp, H., Obata, K. \& Schachner, M. (2000) Recovery of emotional behaviour in neural cell adhesion molecule (NCAM) null mutant mice through transgenic expression of NCAM180. Eur $J$ Neurosci 12, 3291-3306.

Stork, O., Welzl, H., Wotjak, C.T., Hoyer, D., Delling, M., Cremer, H. \& Schachner, M. (1999) Anxiety and increased 5-HT1A receptor response in NCAM null mutant mice. Journal of neurobiology $\mathbf{4 0}$, 343-355.

Touyarot, K. \& Sandi, C. (2002) Chronic restraint stress induces an isoform-specific regulation on the neural cell adhesion molecule in the hippocampus. Neural Plast 9, 147-159.

Touyarot, K., Venero, C. \& Sandi, C. (2004) Spatial learning impairment induced by chronic stress is related to individual differences in novelty reactivity: search for neurobiological correlates. Psychoneuroendocrinology 29, 290-305.

Tsoory, M., Guterman, A. \& Richter-Levin, G. (2008) Exposure to stressors during juvenility disrupts development-related alterations in the PSA-NCAM to NCAM expression ratio: potential relevance for mood and anxiety disorders. Neuropsychopharmacology 33, 378-393.

Varea, E., Castillo-Gomez, E., Gomez-Climent, M.A., Blasco-lbanez, J.M., Crespo, C., Martinez-Guijarro, F.J. \& Nacher, J. (2007) Chronic antidepressant treatment induces contrasting patterns of synaptophysin and PSA-NCAM expression in different regions of the adult rat telencephalon. Eur Neuropsychopharmacol 17, $546-557$.

Venero, C., Herrero, A.I., Touyarot, K., Cambon, K., Lopez-Fernandez, M.A., Berezin, V., Bock, E. \& Sandi, C. (2006) Hippocampal upregulation of NCAM expression and polysialylation plays a key role on spatial memory. Eur J Neurosci 23, 1585-1595.

Venero, C., Tilling, T., Hermans-Borgmeyer, I., Schmidt, R., Schachner, M. \& Sandi, C. (2002) Chronic stress induces opposite changes in the mRNA expression of the cell adhesion molecules NCAM and L1. Neuroscience 115, 1211-1219.

Vyas, A. \& Chattarji, S. (2004) Modulation of different states of anxiety-like behavior by chronic stress. Behav Neurosci 118, $1450-1454$

Vyas, A., Jadhav, S. \& Chattarji, S. (2006) Prolonged behavioral stress enhances synaptic connectivity in the basolateral amygdala. Neuroscience 143, 387-393.

Vyas, A., Mitra, R., Shankaranarayana Rao, B.S. \& Chattarji, S. (2002) Chronic stress induces contrasting patterns of dendritic remodeling in hippocampal and amygdaloid neurons. J Neurosci 22, 6810-6818.

Vyas, A., Pillai, A.G. \& Chattarji, S. (2004) Recovery after chronic stress fails to reverse amygdaloid neuronal hypertrophy and enhanced anxiety-like behavior. Neuroscience 128, 667-673.

Watanabe, Y., Gould, E., Cameron, H.A., Daniels, D.C. \& McEwen, B.S. (1992) Phenytoin prevents stress- and corticosterone-induced atrophy of CA3 pyramidal neurons. Hippocampus 2, 431-435.

Woolley, C.S., Gould, E. \& McEwen, B.S. (1990) Exposure to excess glucocorticoids alters dendritic morphology of adult hippocampal pyramidal neurons. Brain Res 531, 225-231.

\section{Acknowledgments}

The authors thank Melitta Schachner for the initial donation of breeding couples to establish the conditional NCAM-deficient mice colony, as well as Mark Fajans and Coralie Siegmund for their excellent technical assistance. This work has been supported by grants from the EU (FP6-2003-LIFESCIHEALTH-II512012, PROMEMORIA; and FP7-HEALTH-F2M-2008-201600, MemStick), the Swiss National Science Foundation (3100A0108102 and 310000-120791) and intramural funding from the EPFL.

\section{Supporting Information}

Additional Supporting Information may be found in the online version of this article:

Figure S1. NCAM and PSA-NCAM expression levels in the hippocampus of chronically stressed conditional NCAM-deficient mice. No signifcant differences in NCAM (a) and PSA-NCAM (b) expression levels were found in the hippocampus between stressed and control conditional NCAM-deficient (NCAMffcre) mice. Results are the mean \pm SEM ( $n=5 /$ group)

Table S1. Anxiety-like behaviors of chronically stressed and control conditional NCAM-deficient mice in the OF 
Bisaz and Sandi

test. Stressed conditional NCAM-deficient (NCAMffcre) mice visited less frequently, spent less time in the center of the OF test and displayed a higher average distance to the center point compared with control NCAMffcre mice. No difference in general locomotor behavior was found between stressed and control NCAMffcre mice, as evident by similar total distance moved and average velocity. Results are the mean \pm SEM ( $n=6 /$ group, ${ }^{*} P<0.05$ versus controls; * ${ }^{*} P<0.01$ versus controls)

As a service to our authors and readers, this journal provides supporting information supplied by the authors. Such materials are peer-reviewed and may be re-organized for online delivery, but are not copy-edited or typeset. Technical support issues arising from supporting information (other than missing files) should be addressed to the authors. 